\title{
Distribuição Volumétrica e Espectro de Gotas de Pontas de Pulverização de Baixa Deriva ${ }^{1}$
}

\author{
Volumetric Distribution and Droplet Spectrum by Low Drift Spray Nozzles
}

\author{
VIANA, R.G. ${ }^{2}$, FERREIRA, L.R. ${ }^{3}$, FERREIRA, M.C. ${ }^{4}$, TEIXEIRA, M.M. ${ }^{5}$, ROSELL, J.R. ${ }^{6}$, TUFFI \\ SANTOS, L.D. ${ }^{7}$ e MACHADO, A.F.L. ${ }^{8}$
}

\begin{abstract}
RESUMO - Objetivou-se neste trabalho avaliar a distribuição volumétrica e o espectro de gotas das pontas de pulverização de baixa deriva TTI110015, AI110015 e AVI11001 sob diferentes condições operacionais. A distribuição volumétrica foi determinada em bancada de ensaios padronizada analisando o coeficiente de variação (CV\%) de uma barra simulada em computador, utilizando pressões de 200, 300 e $400 \mathrm{kPa}$, altura de 30, 40 e $50 \mathrm{~cm}$ em relação à bancada e espaçamento entre pontas de 40 a $100 \mathrm{~cm}$. O espectro de gotas foi produzido utilizando-se apenas água como calda em um analisador de partículas em meio aquoso, nas pressões de 200, 300 e $400 \mathrm{kPa}$. Foram avaliados o DMV, a porcentagem de gotas com diâmetro inferior a $100 \mu \mathrm{m}(\% 100 \mu \mathrm{m})$ e a amplitude relativa (AR). As pontas proporcionaram perfil descontinuo nas pressões de 300 e $400 \mathrm{kPa}$ e uniforme a $200 \mathrm{kPa}$. Na pressão de $200 \mathrm{kPa}$, as pontas foram adequadas apenas para aplicação em faixa, e a 300 e $400 \mathrm{kPa}$, apenas para área total. Ocorreu menor CV (abaixo de 7\%) com a maior pressão de trabalho e menor espaçamento entre pontas. À medida que se aumentou a pressão de trabalho, reduziu-se o DMV. As pontas TTI1 10015 e AI1 10015 em todas as pressões e a ponta AVI11001 na pressão de $200 \mathrm{kPa}$ produziram gotas extremamente grossas e gotas grossas nas pressões de 300 e $400 \mathrm{kPa}$ apenas para a ponta AVI11001. As pontas proporcionaram baixos valores de amplitude relativa (AR) e gotas de tamanho uniforme, bem como produziram baixa porcentagem de gotas menores que $100 \mu \mathrm{m}$, principalmente TTI1 10015 e AI110015, com menor risco de deriva.
\end{abstract}

Palavras-chave: bico com indução de ar, barra de pulverização, tecnologia de aplicação, deriva.

\begin{abstract}
This study aimed to evaluate the distribution profile and droplet spectra of low drift spray nozzles TTI1 10015, AI1 10015 and AI1 1001 under different operational conditions. The volumetric distribution was determined on a test table (patternator), by analyzing the coefficient of variation (CV) of a computer simulated spraying boom at pressures 200, 300 and $400 \mathrm{kPa}$, height of 30,40 and $50 \mathrm{~cm}$ in relation to the patternator and nozzle spacing from 40 to $100 \mathrm{~cm}$. Volumetric Median Diameter (VMD), span (A.R.) and percentage of droplets below $100 \mu \mathrm{m}$ (\% $100 \mu \mathrm{m}$ ) diameter, at pressures 200, 300 and $400 \mathrm{kPa}$ were evaluated. Nozzles were adequate at pressure $200 \mathrm{kPa}$ only for band application and at pressures 300 and $400 \mathrm{kPa}$ only fortotal area application. Satisfactory CV (under 7\%) was obtained, when highest pressure and lowest space between nozzles were applied. Droplet size (VMD) was reduced as pressure was increased. Nozzles TTI1 10015 and AI1 1015 at all pressures and nozzle AVI1 1001, at $200 \mathrm{kPa}$, presented extra coarse droplets and coarse droplets with AVI1 1001 at pressures 300 and $400 \mathrm{kPa}$. Nozzles AI and TTI presented more uniform droplet size at the highest pressure than nozzle AVI, considering the span value. Nozzles presented a low percentage of droplets below \% $100 \mu \mathrm{m}$, especially nozzles TTI1 10015 and AI1 10015, with reduced drift risk.
\end{abstract}

Keywords: air induction nozzle, spraying boom, application technology, drift.

1 Recebido para publicação em 21.2.2010 e na forma revisada em 15.6.2010.

2 Doutorando em Fitotecnia da Universidade Federal de Viçosa - DFT/UFV, bolsista do CNPq. Avenida PH Holfs, SN. Bairro Centro, <rafaelgomesviana@yahoo.com.br>; ${ }^{3}$ Professor, Dr., do Dep. de Fitotecnia, DFT/UFV; ${ }^{4}$ Professor, Dr., do Dep. de Fitossanidade - UNESP/Jaboticabal; ${ }^{5}$ Professor, Dr., Dep. de Engenharia Agrícola, DEA/UFV; ${ }^{6}$ Professor, Dr., da Universitat de Lleida-Espanha; ${ }^{7}$ Professor, Dr., do Instituto de Ciências Agrárias, UFMG; ${ }^{8}$ Bolsista do Programa Nacional de Pós-Doutorado, Universidade Federal do Tocantins - UFT.

Planta Daninha, Viçosa-MG, v. 28, n. 2, p. 439-446, 2010 


\section{INTRODUÇÃO}

Uma correta tecnologia de aplicação de agrotóxicos visa colocar a quantidade certa de ingrediente ativo no alvo, com a máxima eficiência e da maneira mais econômica possivel, minimizando impactos ao ambiente (Matthews, 2002).

As pontas de pulverização são consideradas os principais componentes da pulverização hidráulica, pois promovem características que asseguram melhor segurança e efetividade no controle de pragas, doenças e plantas daninhas. O sucesso na aplicação de agrotóxico ocorre quando se dispõe de pontas de pulverização que propiciem distribuição transversal uniforme, espectro de gotas semelhante e de tamanho adequado (Cunha, 2003).

É provável que a distribuição uniforme de um determinado diâmetro e o número de gotas possibilitem o sucesso da operação, mesmo que se utilize a aplicação em volume baixo. Nesse caso, cresce a importância de se conhecer a melhor combinação de densidade e diâmetro de gotas, volume e concentração de ingrediente ativo na calda, para as principais pragas, cujo controle é realizado via pulverização (Ferreira, 2003).

A uniformidade de distribuição volumétrica das gotas da calda é uma característica que serve de referência para verificar a distribuição do ingrediente ativo no alvo. Essa uniformidade é diretamente alterada pelo espaçamento entre pontas, pressão de trabalho, altura da barra em relação ao alvo e ângulo de abertura do jato das pontas de pulverização. A uniformidade da distribuição é aferida pelo coeficiente de variação da sobreposição da deposição de um conjunto de pontas em uma barra (Bauer \& Raetano, 2004). A uniformidade de distribuição preconizada pela norma UNE-EN 12761:2 (2002) estabelece que o coeficiente de variação (CV\%) da sobreposição de jatos seja menor que $7 \%$ quando utilizados altura, espaçamento e pressão recomendados pelo fabricante e de até 9\% em configuração distinta.

Distribuição desuniforme de calda, abaixo do volume mínimo exigido, produz controle insuficiente, e quantidades acima causam perdas financeiras, intoxicação nas culturas e danos ao ambiente (Cordeiro, 2001).
A determinação do espectro de gotas produzidas pelas pontas de pulverização é imprescindível para a aplicação de herbicidas. A partir dessa informação, efetua-se a escolha da ponta de acordo com o potencial de deriva, as características do herbicida e os riscos de volatilização e escorrimento de calda nas folhas das plantas daninhas. Segundo Womac et al. (1999), os fatores que influenciam o espectro de gotas produzidas por determinada ponta de pulverização são: vazão nominal, ângulo de descarga, pressão de operação, propriedades da calda e tipo de ponta de pulverização.

Em condições climáticas ótimas, gotas de pequeno diâmetro proporcionam maior densidade de gotas depositadas sobre o alvo. Contudo, aumenta-se o risco de contaminação ambiental por deriva em condições adversas, como temperatura elevada, baixa umidade relativa do ar e alta velocidade de vento (Cross et al., 2001). A utilização de gotas com maior diâmetro diminui o risco de deriva, porém, devido ao seu peso, elas podem não aderir às superficies das folhas e terminar no solo (Teixeira, 1997).

Os parâmetros de maior importância para a determinação da população de gotas são o diâmetro da mediana volumétrica (DMV), a amplitude relativa (AR) e a porcentagem de gotas com diâmetro inferior a $100 \mu \mathrm{m}$. Essas características conjuntamente definem o potencial de deriva de gotas, a homogeneidade de gotas e o tamanho característico das gotas produzidas por um determinado bico de pulverização. Quanto maior o valor da amplitude relativa (AR), maior é a faixa de tamanho das gotas pulverizadas. Espectro de gotas homogêneo tem valor de amplitude relativa que tende a zero. Os valores de DMV e amplitude relativa devem ser analisados conjuntamente para a caracterização da pulverização. Isoladamente, o DMV é um valor de referência e não determina a dispersão dos dados em torno do valor.

A deriva de herbicidas não seletivos em plantações de culturas sensiveis pode resultar em intoxicações e danos às plantas cultivadas, afetar negativamente as características produtivas e reduzir a produtividade da cultura. Tuffi Santos et al. (2007) relatam mudanças no crescimento e na morfoanatomia foliar 
em mudas de eucalipto submetidas à deriva de glyphosate, além de redução do potencial produtivo com sintomas acima de $30 \%$ de intoxicação. Rigoli et al. (2008) observaram danos no desenvolvimento e crescimento em plântulas de beterraba e cenoura; efeito similar foi observado em tomate (Figueredo et al., 2007) e em maracujazeiro-amarelo (Wagner Júnior et al., 2008), todos por efeito da deriva de glyphosate.

Com o intuito de reduzir a deriva de gotas de herbicidas não seletivos, recomendam-se pontas de pulverização com indução de ar para aplicações de herbicidas sistêmicos em pósemergência. Essas pontas também são recomendadas para qualquer herbicida aplicado diretamente ao solo e em pré-emergência (Viana, 2007).

Um dos problemas de pontas de pulverização com indução de ar é que diversas das ofertadas no mercado não possuem informações sobre a população e tamanho de gotas produzidas, o risco potencial de deriva e a distribuição volumétrica. Segundo Viana et al. (2007), essas informações são indispensáveis para a escolha correta da ponta a fim de se obter maior capacidade operacional, eficiência na cobertura do alvo e menor risco ambiental.

Objetivou-se neste trabalho avaliar a distribuição volumétrica e o espectro de gotas das pontas de pulverização de baixa deriva TTI110015, AI110015 e AVI11001, sob diferentes condições operacionais.

\section{MATERIAL E MÉTODOS}

A avaliação da distribuição volumétrica foi realizada no Centre de Mecanitzaciò Agrária de la Genaralitat de Catalunya, no campus Universitário da Universitat de Lleida - España, utilizando as pontas TTI110015, AI $110015 \mathrm{e}$ AVI11001.

Os perfis de distribuição foram determinados utilizando-se uma barra porta-bicos sobre uma mesa de teste para pontas de pulverização hidráulica, composta por canaletas em $\mathrm{V}$, separadas entre si em $5 \mathrm{~cm}$, padronizadas de acordo com a norma ISO 5682 / 1 (ISO, 1986).

Foram utilizadas 10 unidades de cada ponta, instaladas isoladamente no centro da mesa, de modo que o jato fosse lançado na posição vertical. Para cada ponta, realizaramse cinco amostragens. Durante 60 segundos, foi coletado o líquido em provetas graduadas, alinhadas com cada canaleta ao longo da faixa de deposição.

Com base nos volumes médios coletados nas repetições, em cada tratamento, foram determinados os perfis de distribuição volumétrica de cada ponta, com posterior simulação do padrão médio de distribuição volumétrica ao longo da barra de pulverização, o qual foi determinado em software (Microsoft Excel), conforme realizado por Freitas et al. (2005). Trabalhou-se com altura da barra de 30, 40 e $50 \mathrm{~cm}$ em relação à bancada e pressões de 200, 300 e $400 \mathrm{kPa}$. Foram simulados espaçamentos entre pontas de $40,45,50,80,100$ e $120 \mathrm{~cm}$.

Por ocasião da análise do perfil de distribuição volumétrica, foi realizada a coleta de líquido de cada ponta em 60 segundos, para se avaliar a vazão. Os dados de vazão serviram para calcular o volume de calda gasto em uma situação hipotética com deslocamento de $3 \mathrm{~km} \mathrm{~h}^{-1}$ e altura de $50 \mathrm{~cm}$ do alvo.

A análise da população de gotas foi realizada no Laboratório de Análise de Partículas (LAPAR) da Universidade Estadual Paulista, campus de Jaboticabal, com o equipamento Mastersizer S Malvern Instruments Ltd., por difração da luz laser, utilizando apenas a água como líquido pulverizado.

Foram utilizadas cinco pontas de pulverização selecionadas ao acaso em um lote de 10 , representando cada ponta uma repetição, em um delineamento inteiramente casualizado.

O feixe laser foi posicionado a $350 \mathrm{~mm}$ abaixo da ponta de pulverização, para se analisar em duas dimensões através do plano horizontal. Cada ponta foi instalada em um transportador radial, o que permitiu que o jato pulverizado passasse transversalmente através do feixe luminoso em três segundos. Foram utilizadas as pressões de 200, 300 e $400 \mathrm{kPa}$, proporcionadas por meio de um pulverizador pressurizado a ar comprimido (pressão constante). Foram determinados o diâmetro da mediana volumétrica (DMV), a amplitude relativa (AR) e a porcentagem de gotas com diâmetro inferior a $100 \mu \mathrm{m}$. 
A análise da distribuição volumétrica das pontas de pulverização foi realizada pelo cálculo do coeficiente de variação $(\mathrm{CV} \%)$ da sobreposição dos jatos; os dados de espectro de gotas e vazão foram submetidos à ANOVA, e as médias, comparadas pelo teste de Tukey a $5 \%$ de probabilidade.

\section{RESULTADOS E DISCUSSÃO}

As pontas avaliadas nas pressões de $300 \mathrm{e}$ $400 \mathrm{kPa}$ proporcionaram perfis de distribuição
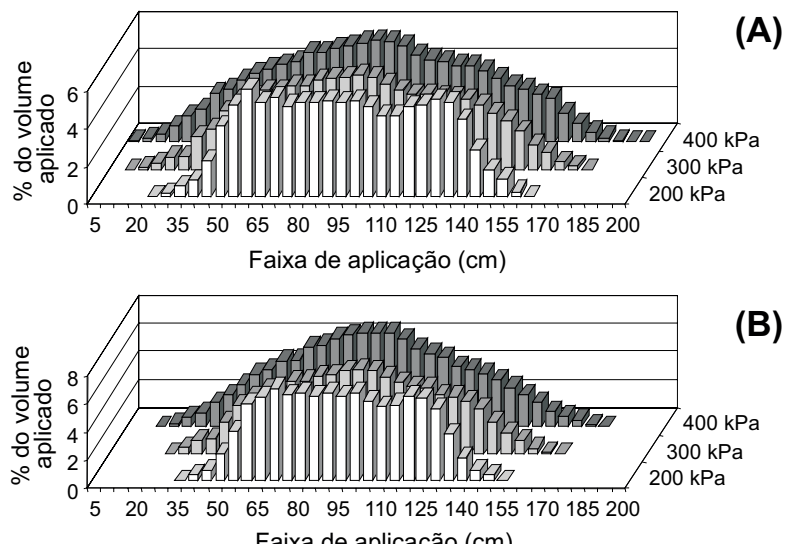

Faixa de aplicação $(\mathrm{cm})$

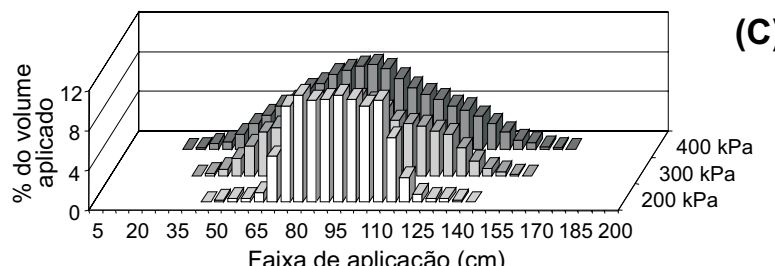

Faixa de aplicação $(\mathrm{cm})$

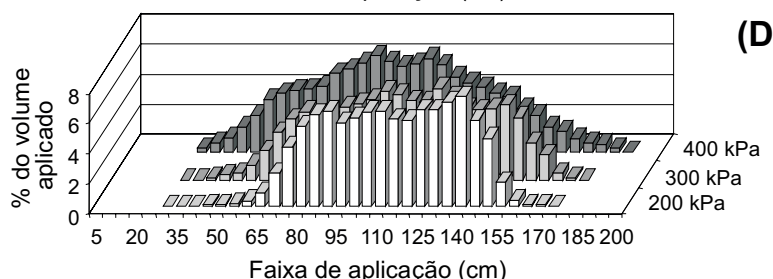

(D)

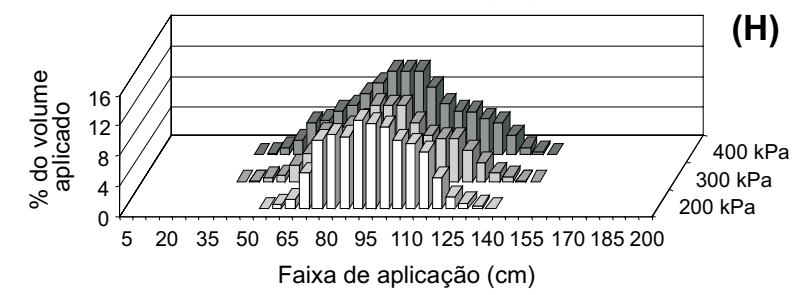

(I)

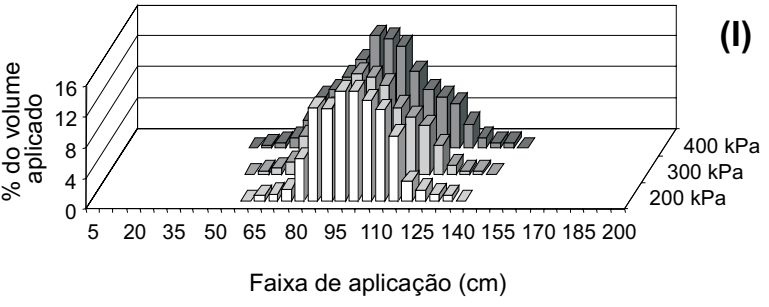

$200 \mathrm{kPa} \square 300 \mathrm{kPa} \square 400 \mathrm{kPa}$

Figura 1 - Perfis de distribuição das pontas TTI110015 a 50 (A), 40 (B) e $30 \mathrm{~cm}$ de altura (C), AI110015 a 50 (D), 40 (E) e $30 \mathrm{~cm}$

de altura (F) e AVI11001 a $50(\mathrm{G}), 40(\mathrm{H})$ e $30 \mathrm{~cm}$ de altura (I), operando nas pressões de 200, 300 e $400 \mathrm{kPa}$.

Planta Daninha, Viçosa-MG, v. 28, n. 2, p. 439-446, 2010

descontínuos, com maior acúmulo de líquido na parte central e decréscimo gradual nas extremidades (Figura 1). Pontas com esse perfil de distribuição são indicadas para aplicação em área total, com sobreposição de jatos (Matuo et al., 2001). Na pressão de $200 \mathrm{kPa}$, observa-se perfil de distribuição uniforme, com depósito de líquido semelhante na parte central e decréscimo abrupto nas extremidades. Pontas com esse perfil são indicadas para aplicação em faixa, sem a sobreposição de jatos (Matuo et al., 2001).
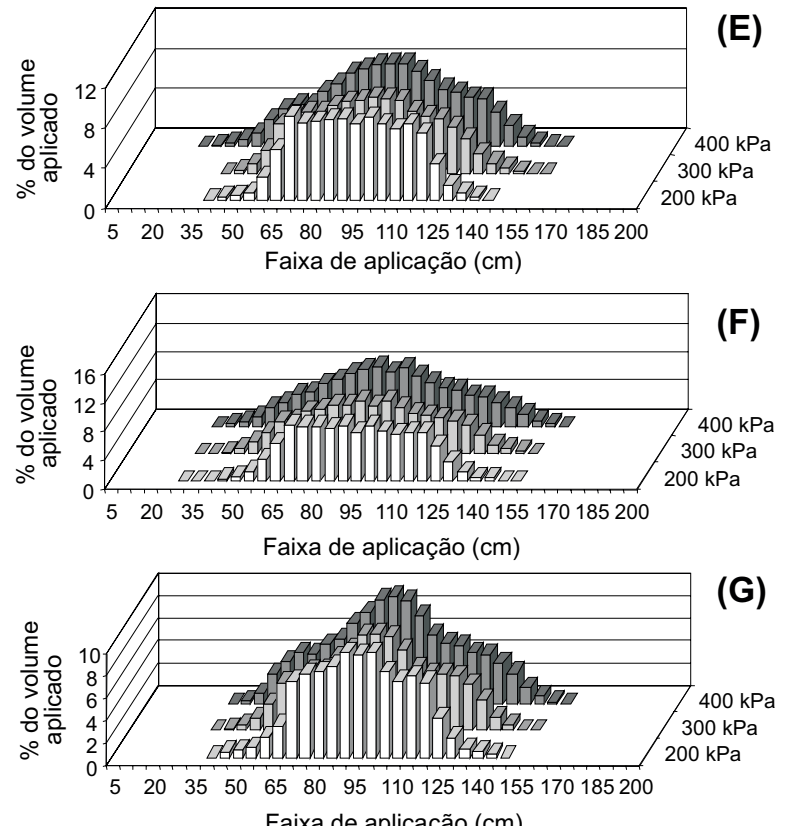

(H) 
Comportamentos semelhantes foram verificados por Viana et al. (2007) e Freitas et al. (2005), ao avaliarem o perfil de distribuição de pontas de jato plano. Na menor pressão avaliada $(100 \mathrm{kPa})$ esses autores constataram perfis uniformes, com maiores $\mathrm{CV}(\%)$ quando da sobreposição de jato das pontas. Esse comportamento é explicado em razão do menor ângulo do jato na pressão utilizada, o que resulta em menor faixa de aplicação e menor deslocamento das gotas para as extremidades. Por outro lado, com o incremento na pressão aumenta-se a faixa de aplicação e há maior deslocamento das gotas para as extremidades, devido ao aumento no ângulo do jato e redução no tamanho de gotas.

A faixa de aplicação, em todas as situações, foi reduzida na menor altura de barra em relação ao alvo.

Para a uniformidade de distribuição ao longo da barra, verifica-se que apenas a ponta TTI 110015 a 50, 40 e $30 \mathrm{~cm}$ de altura e espaçamento entre pontas de 50,40 e $45 \mathrm{~cm}$, respectivamente, é indicada para aplicação em área total a $200 \mathrm{kPa}$ (Tabela 1). No entanto, se houver variações na altura da barra (trepidação), a distribuição proporcionada por essa ponta, nessas condições, pode se tornar desuniforme. Variações na altura da barra são muito comuns em aplicações em florestas de eucalipto, devido à irregularidade do terreno ou a obstáculos como tocos e resíduos, da cultura anterior. $\mathrm{O}$ alto $\mathrm{CV}$ da sobreposição dos jatos deve-se ao perfil de distribuição individual das pontas, que não favorece a sobreposição de jatos.

$\mathrm{Na}$ pressão de $300 \mathrm{kPa}$ (Tabela 1), a ponta TTI 110015 pode ser utilizada nos espaçamentos entre pontas de 45 e $50 \mathrm{~cm}$ em todas as alturas avaliadas. Destacam-se as alturas de 40 e $30 \mathrm{~cm}$ como uma maneira de reduzir a deriva de gotas sem, contudo, influenciar a distribuição de calda no alvo.

A ponta AI110015 a $300 \mathrm{kPa}$ (Tabela 1) proporcionou distribuição uniforme a $50 \mathrm{~cm}$ do alvo no espaçamento entre pontas de $50 \mathrm{~cm}$; na altura de $40 \mathrm{~cm}$ e espaçamento de $40 \mathrm{~cm}$; e na altura de $30 \mathrm{~cm}$ com espaçamento de $45 \mathrm{~cm}$. Para ponta AVI11001 na mesma pressão, é recomendado espaçamento entre pontas de 45 e $50 \mathrm{~cm}$ na altura de $50 \mathrm{~cm}$ e espaçamento entre pontas de $45 \mathrm{~cm}$ na altura de $40 \mathrm{~cm}$.

Tabela 1 - Coeficiente de variação (CV\%) do perfil de distribuição das pontas de pulverização TTI 110015, AI 110015 e AVI 11001 nas pressões de 200, 300 e $400 \mathrm{kPa}$ a 50, 40 e $30 \mathrm{~cm}$ de altura de barra, espaçados em 40, 45, 50, 80, 90 e $100 \mathrm{~cm}$ na barra de pulverização

\begin{tabular}{|c|c|c|c|c|c|c|c|c|c|c|}
\hline \multirow{4}{*}{$\begin{array}{c}\text { Pressão } \\
(\mathrm{kPa})\end{array}$} & \multirow{4}{*}{$\begin{array}{l}\text { Espaçamento entre } \\
\text { pontas }(\mathrm{cm})\end{array}$} & \multicolumn{9}{|c|}{ Coeficiente de Variação (CV\%)* } \\
\hline & & \multicolumn{3}{|c|}{ TTI 110015} & \multicolumn{3}{|c|}{ AI 110015} & \multicolumn{3}{|c|}{ AVI 11001} \\
\hline & & \multicolumn{9}{|c|}{ Altura da barra $(\mathrm{cm})$} \\
\hline & & 50 & 40 & 30 & 50 & 40 & 30 & 50 & 40 & 30 \\
\hline \multirow{6}{*}{200} & 40 & 13 & 4 & 15 & 8 & 18 & 14 & 11 & 15 & 17 \\
\hline & 45 & 9 & 8 & 5 & 15 & 21 & 20 & 14 & 9 & 29 \\
\hline & 50 & 4 & 16 & 13 & 24 & 22 & 22 & 14 & 10 & 40 \\
\hline & 80 & 26 & 8 & 63 & 10 & 34 & 24 & 38 & 62 & 86 \\
\hline & 90 & 18 & 16 & 90 & 12 & 56 & 46 & 60 & 88 & 109 \\
\hline & 100 & 23 & 31 & 92 & 39 & 65 & 55 & 67 & 90 & 112 \\
\hline \multirow{6}{*}{300} & 40 & 39 & 37 & 37 & 11 & 5 & 37 & 57 & 56 & 56 \\
\hline & 45 & 5 & 7 & 2 & 10 & 8 & 4 & 5 & 6 & 12 \\
\hline & 50 & 7 & 4 & 6 & 7 & 12 & 8 & 3 & 10 & 17 \\
\hline & 80 & 14 & 11 & 12 & 19 & 14 & 10 & 21 & 39 & 66 \\
\hline & 90 & 15 & 9 & 25 & 14 & 32 & 24 & 35 & 59 & 86 \\
\hline & 100 & 12 & 11 & 37 & 12 & 46 & 37 & 49 & 67 & 92 \\
\hline \multirow{6}{*}{400} & 40 & 50 & 47 & 47 & 2 & 8 & 48 & 73 & 71 & 70 \\
\hline & 45 & 1 & 3 & 5 & 3 & 6 & 5 & 12 & 6 & 10 \\
\hline & 50 & 3 & 5 & 3 & 4 & 5 & 4 & 7 & 10 & 16 \\
\hline & 80 & 6 & 5 & 11 & 6 & 13 & 10 & 19 & 39 & 58 \\
\hline & 90 & 7 & 6 & 20 & 6 & 21 & 19 & 27 & 59 & 76 \\
\hline & 100 & 9 & 9 & 30 & 9 & 34 & 29 & 37 & 67 & 83 \\
\hline
\end{tabular}

* Valores abaixo de 7\% são considerados satisfatórios. 
As poucas opções de configurações de espaçamento entre pontas e altura de barra para essas pontas podem estar relacionadas ao menor ângulo de pulverização. Isso ocorre, provavelmente, devido ao mecanismo de a ponta perder energia cinética das gotas, provocada pela indução de ar. Esse fato é relatado por Viana et al. (2007), que indicaram a possibilidade de perda de carga devido à indução de ar das pontas avaliadas, sendo necessário maior pressão de trabalho para que ocorra melhora na distribuição da calda. Ferreira et al. (2007) avaliaram a ponta ADGA 110015 e verificaram que o perfil de distribuição do jato foi simétrico nas pressões de trabalho de 207 e $310 \mathrm{kPa}$. Verificaram também que na maior pressão a barra poderia operar com menor altura ou com as pontas mais espaçadas entre si. Isso porque o maior ângulo de aspersão do jato resultou em diminuição do coeficiente de variação. Entretanto, na maior pressão houve redução significativa no diâmetro das gotas, aumentando o potencial de cobertura do alvo, bem como a suscetibilidade à deriva e à evaporação.

A maior quantidade de configurações da ponta TTI 110015 na pressão de $300 \mathrm{kPa}$, em detrimento das demais pontas (Tabela 1), devese ao mecanismo de formação do jato. Nesse caso, há aumento no ângulo devido ao impacto dele em um plano inclinado e, por consequência, aumenta-se a sobreposição dos jatos. Viana et al. (2009) e Freitas et al. (2005) observaram que, mesmo em baixas pressões, as pontas de pulverização com sistema de impacto podem ser utilizadas em espaçamentos de até $1,0 \mathrm{~m}$, o que favorece a redução no volume de calda e o aumento no rendimento operacional.

A pressão de $400 \mathrm{kPa}$ (Tabela 1) proporcionou maior número de configurações adequadas à aplicação em área total para todas as pontas, em razão do aumento no ângulo do jato e, consequentemente, aumento na sobreposição de jato. A ponta TTI 110015 a 50 e $40 \mathrm{~cm}$ do alvo proporcionou melhor distribuição nos espaçamentos entre 45 e $90 \mathrm{~cm}$. Para a altura de $30 \mathrm{~cm}$, os melhores espaçamentos foram de 45 e $50 \mathrm{~cm}$. As configurações encontradas são excelentes para duas ocasiões. Nas alturas de 50 e $40 \mathrm{~cm}$ em relação ao alvo, pode-se aumentar o espaçamento entre pontas e, dessa maneira, reduzir o volume de caldo gasto e o número de passadas do pulverizador por área e aumentar o rendimento operacional dos pulverizadores. Fato semelhante foi observado por Freitas et al. (2005) ao utilizarem a ponta TT11002, que pode ser combinada com espaçamentos de até $120 \mathrm{~cm}$ e resulta em excelente distribuição e baixo volume de calda. Além disso, pode-se diminuir a altura da ponta TTI 110015 para $30 \mathrm{~cm}$ e, assim, reduzir a distância da gota em relação ao alvo; dessa forma, reduz-se a deriva de gotas, porém com menor espaçamento e maior gasto de calda.

A ponta AI110015 na pressão de $400 \mathrm{kPa}$ (Tabela 1) apresentou boa uniformidade na altura de $50 \mathrm{~cm}$ e espaçamento entre pontas de 40 a $90 \mathrm{~cm}$; e na altura de 40 e $30 \mathrm{~cm}$ e espaçamento de 45 e $50 \mathrm{~cm}$.

Observa-se na Tabela 2 aumento da vazão com o incremento na pressão. Quando se comparam as pontas na mesma pressão, observase que a ponta AVI11001 apresenta menor vazão, em concordância com as informações dos fabricantes.

As pontas proporcionaram tamanho de gotas semelhante e o mesmo comportamento. Ầ medida que se aumentou a pressão, reduziuse o tamanho de gota (Tabela 3).

O DMV (Tabela 3) proporcionado pelas pontas TTI 110015 e AI110015 em todas as pressões e pela ponta AVI11001 na pressão de $200 \mathrm{kPa}$ classifica as gotas como extremamente grossas e como gotas grossas para a ponta AVI11001 nas pressões de 300 e $400 \mathrm{kPa}$, segundo a classificação da British Crop Protection Council (Doble et al., 1985). O motivo do menor tamanho de gota gerado pela ponta AVI11001, em comparação com as

Tabela 2 - Vazão das pontas de pulverização TTI110015, AI110015 e AVI11001 nas pressões de 200, 300 e $400 \mathrm{kPa}$

\begin{tabular}{|c|c|c|c|}
\hline \multirow{2}{*}{$\begin{array}{c}\text { Pressão } \\
(\mathrm{kPa})\end{array}$} & \multicolumn{4}{|c|}{ Vazão $\left(\mathrm{L} \mathrm{min}^{-1}\right)$} \\
\cline { 2 - 4 } & \multicolumn{4}{|c|}{ Ponta } \\
\cline { 2 - 4 } & TTI110015 & AI110015 & AVI11001 \\
\hline 200 & $0,487 \mathrm{~A} \mathrm{c}$ & $0,520 \mathrm{~A} \mathrm{c}$ & $0,332 \mathrm{~B} \mathrm{c}$ \\
\hline 300 & $0,622 \mathrm{~A} \mathrm{~b}$ & $0,600 \mathrm{~A} \mathrm{~b}$ & $0,375 \mathrm{~B} \mathrm{~b}$ \\
\hline 400 & $0,694 \mathrm{~A} \mathrm{a}$ & $0,696 \mathrm{~A} \mathrm{a}$ & $0,437 \mathrm{~B} \mathrm{a}$ \\
\hline
\end{tabular}

Médias seguidas de mesmas letras maiúsculas, na linha, e minúsculas, na coluna, não diferem significativamente a $5 \%$ de probabilidade pelo teste de Tukey. 
Tabela 3 - Diâmetro da mediana volumétrica (DMV), amplitude relativa (AR) e porcentagem do volume em gotas menores que $100 \mu \mathrm{m}(\% 100)$ das pontas de pulverização TTI110015, AI110015 e AVI11001, nas pressões de 200, 300 e $400 \mathrm{kPa}$

\begin{tabular}{|c|c|c|c|c|}
\hline Ponta & $\begin{array}{c}\text { Pressão } \\
(\mathrm{kPa})\end{array}$ & $\begin{array}{c}\mathrm{DMV}^{*} \\
(\mathrm{~mm})\end{array}$ & $\mathrm{AR}$ & $\begin{array}{c}\% 100 \\
(\mathrm{~mm})\end{array}$ \\
\hline \multirow{3}{*}{ TTI 110015} & 200 & 919 a A & 1,43 a $\mathrm{A}$ & 0,16 a B \\
\hline & 300 & $733 \mathrm{~b} \mathrm{~A}$ & 1,37 a A & 0,53 a C \\
\hline & 400 & $692 \mathrm{~b} \mathrm{~A}$ & 1,57 a $B$ & 1,10 a C \\
\hline \multirow{3}{*}{ AI 110015} & 200 & 769 a A & 1,18 a A & $1,41 \mathrm{~b} \mathrm{AB}$ \\
\hline & 300 & $711 \mathrm{ab} A$ & 1,44 a A & $2,57 \mathrm{ab} \mathrm{B}$ \\
\hline & 400 & $570 \mathrm{~b} \mathrm{~A}$ & 1,60 a B & 3,55 a B \\
\hline \multirow{3}{*}{ AVI 11001} & 200 & 478 a B & $2,12 \mathrm{~b} \mathrm{~A}$ & $2,64 \mathrm{c} \mathrm{A}$ \\
\hline & 300 & 413 a B & $2,27 \mathrm{~b} \mathrm{~A}$ & $4,81 \mathrm{~b} \mathrm{~A}$ \\
\hline & 400 & 317 a B & 4,31 a $\mathrm{A}$ & 7,27 a A \\
\hline
\end{tabular}

Letras minúsculas comparam as três pressões dentro de uma mesma ponta; letras maiúsculas comparam a mesma pressão entre as três pontas. Médias comparadas pelo teste de Tukey a 5\% de probabilidade, $*$ gota grossa $=250$ a $375 \mu \mathrm{m}$; gota muito grossa $=$ 375 a $450 \mu \mathrm{m}$; gota extremamente grossa $=>450 \mu \mathrm{m}$,

demais pontas avaliadas, é a baixa vazão desta ponta (menor orifício), o que provoca maior fragmentação do líquido e menor tamanho de gota.

Gotas extremamente grossas são indicadas para aplicação de herbicidas em pré-emergência e herbicidas sistêmicos em pós-emergência, como o glyphosate, nas culturas de eucalipto e café. Essas gotas também são indicadas para aplicações em outras atividades, como a dessecação em condições desfavoráveis, como baixa umidade relativa, alta temperatura e vento (Viana et al., 2007). Entretanto, dependendo de fatores inerentes à constituição química e física da folha de algumas plantas daninhas, existe o risco de escorrimento das gotas para fora do alvo. A ponta de pulverização que promoveu gotas grossas (AVI11001) nas pressões de 300 e $400 \mathrm{kPa}$ (Tabela 4) resultaria em melhor cobertura do alvo, porém são mais propensas à deriva, razão pela qual se deve levar em conta a cobertura necessária e também as condições ambientais.

Observaram-se os menores valores de amplitude relativa para a menor pressão de trabalho utilizada (Tabela 3), com boa uniformidade nos conjuntos de gotas. A mesma tendência foi observada por Fernandes et al. (2007) quando avaliaram pontas de pulverização de jato plano. Destacam-se como mais homogêneas as pontas TTI110015 e AI110015.

A porcentagem de gotas com diâmetro inferior a $100 \mu \mathrm{m}$ (Tabela 3) para as três pontas nas pressões avaliadas está abaixo do padrão para risco de deriva. Segundo Cunha (2003), valores abaixo de $15 \%$ de gotas com diâmetro inferior a $100 \mu \mathrm{m}$ são adequados para aplicações seguras, com menor risco de deriva. A ponta TTI 110015 proporcionou as menores porcentagens de gotas com diâmetro inferior a $100 \mu \mathrm{m}$ - por isso é a mais indicada em locais onde a deriva de gotas deve ser evitada. A ponta AVI11001 proporcionou as maiores porcentagens de gotas com diâmetro inferior a $100 \mu \mathrm{m}$, motivo pelo qual apresenta maior risco de deriva.

As pontas avaliadas devem ser utilizadas para aplicação em área total com pressões de 300 e $400 \mathrm{kPa}$ e em aplicação dirigida na pressão de $200 \mathrm{kPa}$. Elas promovem gotas com baixo potencial de deriva, sendo classificadas de grossas a extremamente grossas. Conforme a classificação das gotas, as pontas podem ser utilizadas para aplicação de herbicidas sistêmicos em pós-emergência e herbicidas em pré-emergência.

\section{LITERATURA CITADA}

BAUER, F. C.; RAETANO, C. G. Distribuição volumétrica de calda produzida pelas pontas de pulverização XR, TP e TJ sob diferentes condições operacionais. Planta Daninha, v. 22 , n. 2 , p. $275-284,2004$

CORDEIRO, A. M. C. Como a tecnologia de aplicação de produtos fitossanitários pode contribuir para o controle de pragas, doenças e plantas daninhas. In: ZAMBOLIM, L. Manejo integrado: fitossanidade, cultivo protegido, pivô central e plantio direto. Viçosa, MG: Universidade Federal de Viçosa, 2001. p. 683-721

CROSS, J. V. et al. Spray deposits and losses in different sized apple trees from an axial fan orchard sprayer: 2. Effects of spray quality. Crop Protec., v. 20, n. 2, p. 333-343, 2001.

CUNHA, J. P. A. R. Tecnologia de aplicação do chlorothalonil no controle de doenças do feijoeiro. 2003. $81 \mathrm{f}$. Tese (Doutorado em Mecanização Agrícola) Universidade Federal de Viçosa, Viçosa, MG, 2003.

DOBLE, S. J. et al. A system for classifying hydraulic nozzles and other atomizers into categories of spray quality. Brit. Crop Protec. Coun., v. 9, n. 1 p. 112-122, 1985. 
FERNANDES, A. P. et al. Caracterização do perfil de deposição e otimização do espaçamento entre bicos na barra de pulverização. Eng. Agríc., v. 27, n. 3, p. 728-733, 2007.

FERREIRA, M. C. Caracterização da cobertura de pulverização necessária para controle do ácaro Brevipalpus phoenicis (G., 1939) em citros. 2003. 64 f. Tese (Doutorado em Produção Vegetal) - Universidade Estadual Paulista, Jaboticabal, 2003

FERREIRA, M. C. et al. Fatores qualitativos da ponta de energia hidráulica ADGA 110015 para pulverização agrícola. Eng. Agríc., v. 27, n. 2, p. 471-478, 2007.

FIGUEREDO, S. S. et al. Influência de doses reduzidas do glyphosate no tomateiro (Lycopersicon esculentum Mill.). Planta Daninha, v. 25, n. 3, p. 849-857, 2007.

FREITAS, F.C.L. et al. Distribuição volumétrica de pontas de pulverização turbo teejet 11002 em diferentes condições operacionais. Planta Daninha, v. 23, n. 1, p. 161-167, 2005.

INTERNATIONAL ORGANIZATION FOR STANDARDIZATION - ISO. Equipment for crop protection - Spraying equipment - Part 2: test methods for agricultural sprayers. Geneva: 1986. 5 p. (ISO 5682/2).

MATTHEWS, G. A. The application of chemicals for plant disease control. In: WALLER, J.M.; LENNÉ, J.M.; WALLER, S.J. Plant pathologist's pocketbook. London: CAB, 2002. p. 345-353.

MATUO, T. et al. Tecnologia de aplicação e equipamentos. In: ABEAS - Curso de proteção de plantas. Módulo 2. Brasília, DF: ABEAS; Viçosa, MG: UFV, 2001. 85 p.
TEIXEIRA, M. M. Influencia del volumen de caldo y de la uniformidad de distribución transversal sobre la eficacia de la pulverización hidráulica. 1997. 310 f. Tese (Doutorado em Agronomia) - Escuela Técnica Superior de Ingenieros Agrônomos, Universidad Politécnica de Madrid, Madrid, 1997.

RIGOLI, R. P. et al. Resposta de plantas de beterraba (Beta vulgaris) e de cenoura (Daucus carota) à deriva simulada de glyphosate. Planta Daninha, v. 26, n. 2, p. 451-456, 2008.

TUFFI SANTOS, L. D. et al. Crescimento do eucalipto sob efeito da deriva de glyphosate. Planta Daninha, v. 25, n. 1, p. 133-137, 2007.

UNE-EN. Maquinaria agrícola y forestal - Pulverizadores y distribuidores de fertilizantes líquidos. Protección medioambiental. Parte 2: Pulverizadores hidráulicos de barras para cultivos bajos. 2002. (UNE-EN 12761-2:2002)

VIANA, R. G. et al. Características técnicas de pontas de pulverização LA-1JC e SR-1. Planta Daninha, v. 25, n. 1, p. 211-218, 2007.

VIANA, R. G. et al. Deposición transversal de líquido de las boquillas de doble abanico TTJ60-11004 y TTJ60-11002 en distintas condiciones operacionales. Planta Daninha, v. 27, n. 2, p. 397-403, 2009.

WAGNER JÚNIOR, A. et al. Deriva simulada de formulações comerciais de glyphosate sobre maracujazeiro-amarelo.

Planta Daninha, v. 26, n. 3, p. 677-683, 2008.

WOMAC, A. R.; MAYNARD, R. A.; KIRK, I. W. Measurement variations in reference sprays for nozzle classification. Trans. ASAE, v. 42, n. 3, p. 609-616, 1999 